\title{
Decolorization of hair dye by lignocellulosic waste materials from contaminated waters
}

\author{
Habib Zahir ${ }^{1}$, Michelle Naidoo ${ }^{1}$, Rada-Mayya Kostadinova ${ }^{1}$, Karla A. Ortiz ${ }^{2}$, Rosario Sun-Kou ${ }^{3}$ and \\ Abel E. Navarro ${ }^{1 *}$
}

' Science Department, Borough of Manhattan Community College, The City University of New York, New York, NY, USA

${ }^{2}$ Department of Chemistry, College of Arts and Science, New York University, New York, NY, USA

${ }^{3}$ Sección Química, Departamento de Ciencias, Pontificia Universidad Católica del Perú, Lima, Perú

Edited by:

Aziz Ullah, Kohat University of

Science and Technology, Pakistan

Reviewed by:

Urs Jans, City College of the City

University of New York, USA

Francesco Di Natale, Università di

Napoli Federico II, Italy

\section{*Correspondence:}

Abel E. Navarro, Science

Department, Borough of Manhattan

Community College, 199 Chambers

Street, New York, NY 10007, USA

e-mail: anavarro@bmcc.cuny.edu
Basic yellow 57 (BY57) was chosen as a model hair dye due to its prevalence in cosmetics wastewaters. This study proposes the use of lignocellulosic materials like spent tea leaves of peppermint (PM), chai tea (CT), and chamomile (CM) as raw adsorbents for the removal of BY57 from contaminated solutions. Batch adsorption experiments were carried out at room temperature to achieve the maximum adsorption capacity. Results indicate that the highest removal is achieved at $\mathrm{pH} 6-8$, with a minimum adsorbent mass of $75 \mathrm{mg}$ and in the absence of salinity, crowding agents and heavy metals. Adsorption equilibria were modeled according to the Langmuir and Freundlich isotherm theories and reported the following trend: PM $>C T>C M$, reaching $q_{\max }$ values of 105,80 , and $38 \mathrm{mg}$ of dye per gram of adsorbent, respectively. Desorption experiments showed that diluted solution of $\mathrm{HCl}$ is able to desorb up to $80 \%$ of the dye and recover the adsorbent to be used in consecutive cycles. Finally, the adsorbents were characterized by Scanning electron microscopy (SEM), indicating that the adsorbents have a porous and heterogeneous surface, showing pockets and protrusions that are potential adsorption sites for the dye.

Keywords: adsorption, basic yellow 57, tea leaves, $\mathrm{pH}$, desorption, isotherm

\section{INTRODUCTION}

Different substances are currently used as coloring agents. They can be divided into two big groups, dyes, and pigments that are soluble and insoluble in water, respectively. Dyes can be defined as substances that confer color without being affected by factors like light, water, and surfactants. Their structure is formed by more than one type of functional groups, mainly aromatic rings that can be nitro-, amino-, and hydroxyl-substituted (Singh-Rai et al., 2005). Since 1856, more than 10,000 types of colorants have been produced. Today, more than $7 \times 10^{5}$ Tons of dyes are produced per year, and more than $10 \%$ is lost during the industrial dyeing process (Zollinger, 1991; Easton, 1995).

Dyes contain chromophores of high molar mass that accumulate in lakes and bays; and promote a decrease in the photosynthetic activity. This also causes a decrease in the available oxygen content, and favors eutrophication processes due to the increase of organic matter (Robinson et al., 2001).

There are several types of chemical and physical processes for the removal of dyes, such as ozonation, oxidative degradation, photochemical processes, bleaching, electrochemical degradation, adsorption, treatment with activated carbon, microfiltration, ionic exchange, irradiation, amongst others (Volesky, 2004; Liu and Wang, 2009; Navarro et al., 2013). Although these techniques have been conventionally used for the removal of dyes and other pollutants; they still show important disadvantages such as high operative costs and generation of byproducts that need further treatment (Robinson et al., 2001; Kotrba et al., 2011; Kim et al., 2013).

Biological treatment by activated sludge has been successfully used for the treatment of dye-containing wastewaters and is widely used in water purification plants. Bioaccumulation has also been applied to the decontamination of pollutants, by using microorganisms such as bacteria, algae, yeast, and fungi (Guo et al., 2008; Gonzalez and Fuentes, 2009; Kotrba et al., 2011). The most important advantage of microorganisms is their selectivity in the uptake of pollutants. However, they also show drawbacks like adequate temperature, enough nutrients, and constant agitation for their operation (Liu and Wang, 2009; Kotrba et al., 2011). Conversely, biosorption utilizes dead biomass like spent tea leaves, dead algae, crustacean shells, eggshells, and other biological residues to eliminate pollutants. The removal is through adsorption that is mainly driven by intermolecular interactions like electrostatic, hydrogen bonding, dipole dipole, and dispersion forces (Liu and Wang, 2009; Iparraguirre et al., 2010; Kim et al., 2013; Navarro et al., 2013). Spectroscopic studies have shown that these biosorbents have active adsorption sites such as carboxyl, hydroxyl, and amino groups on their surfaces. It has been demonstrated that all these groups have a great affinity toward pollutants like heavy metals (Cuizano et al., 2009; Diaz et al., 2013), phenols (Navarro et al., 2008a,b), and dyes (Naidoo et al., 2013; Navarro et al., 2013).

Basic yellow 57 (BY57) hair dye is commonly used in cosmetics in the formulation of hair coloring pastes. As shown in Figure 1, it 


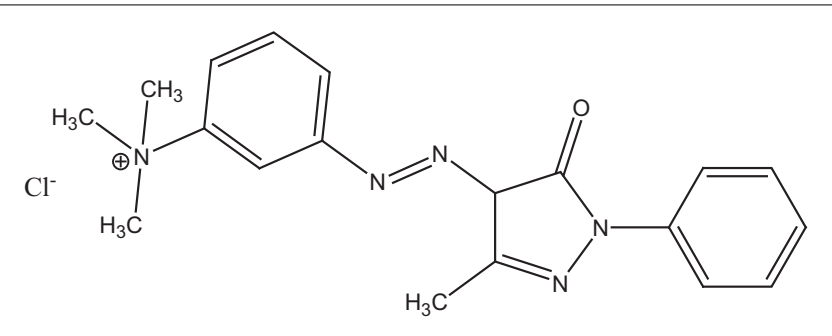

FIGURE 1 | Chemical structure of the cationic dye Basic Yellow 57 (BY57).

is a cationic dye with polar groups like amide, quaternary amine, azo, and heterocyclic rings. BY57 is water soluble at any $\mathrm{pH}$ and in the presence of other organic and inorganic solutes.

The purpose of this study is the use of lignocellulosic waste materials like spent peppermint (PM), chai tea (CT), and chamomile (CM) teas on the removal of BY57 from contaminated solutions. These spent teas are massively discarded by tea-based industries and become a waste disposal concern. We propose that this industrial waste can be recycled and used as ecofriendly adsorbents. Our long term goal envisions the preparation of recyclable filters in continuous-flow industrial processes for the removal of BY57 and other dyes and organic pollutants.

\section{MATERIALS AND METHODS DYE SOLUTIONS}

Stock solution of BY57 was prepared by dissolving the dye in deionized water and brought to a concentration of $1000 \mathrm{mg} / \mathrm{L}$. BY57 dye was donated by Vanshi Chemicals Pvt Ltd. (India). Solutions of varying concentrations were prepared by dilution of the stock with deionized water. The initial $\mathrm{pH}$ of the solutions were measured with a pH-meter (Fisher Scientific, Accumet $\mathrm{AB} 15)$ and adjusted to the required $\mathrm{pH}$ value by adding aliquots of concentrated $\mathrm{HCl}$ and $\mathrm{NaOH}$ solutions prior contact with the adsorbents.

\section{PREPARATION OF THE LIGNOCELLULOSIC ADSORBENTS}

$\mathrm{PM}, \mathrm{CT}$, and CM teabags were purchased from a local supermarket and spent in sequential rinses of boiling distilled water to extract color, smell, and taste. Final rinses were done with boiling deionized water to remove any residual impurity. Then, the teabags were cut open and the adsorbents were oven-dried at a temperature no higher than $50^{\circ} \mathrm{C}$ to prevent any chemical of thermal decomposition. Adsorbents were not modified in their particle size, as they showed appropriate dimensions for their direct use in adsorption experiments. Finally, all adsorbents were stored in plastic containers at room temperature till their usage. No evidence of biological decomposition was observed.

\section{DISCONTINUOUS ADSORPTION EXPERIMENTS}

Batch experiments were carried out in duplicate at room temperature by combining variable masses of the adsorbents with $50 \mathrm{~mL}$ of dye solutions under orbital agitation at $250 \mathrm{rpm}$ for $24 \mathrm{~h}$. Preliminary studies indicated that less than $24 \mathrm{~h}$ are needed to reach equilibrium. Initial solution $\mathrm{pH}$ was adjusted depending on the type of experiment. Adsorption mixture was sealed with parafilm to avoid evaporation. Upon equilibrium, the suspensions were separated by gravity and the remaining dye concentration was determined by spectrophotometry, using a microplate reader (Biotek, Synergy4) at a wavelength of $380 \mathrm{~nm}$. Equilibrium parameters were sequentially optimized, including $\mathrm{pH}$, mass of adsorbent, initial dye concentration, ionic strength, and presence other pollutants like heavy metals (copper, lead, and zinc divalent ions). After the optimization of each parameter, they were directly used for the following experiments. Lead, zinc, and copper salts were utilized in their nitrate forms (Fisher Scientific, reagent grade). Polyethylene glycol $(\mathrm{PEG})(\mathrm{MW}=1000 \mathrm{~g} / \mathrm{mol})$ was also used as an interfering substance and used without further purification (Spectrum Chemicals, reagent grade). Crowding, salinity, and presence of heavy metal ions were carried using the optimum $\mathrm{pH}$, the optimum masses and a initial dye concentration of $100 \mathrm{mg} / \mathrm{L}$.

\section{DESORPTION EXPERIMENTS}

A big-scale adsorption experiment was carried out with all the optimized equilibrium parameters to obtain dye-loaded adsorbents. Then, a known mass of the adsorbent was mixed with $20 \mathrm{~mL}$ of different co-solvents. These co-solvents were chosen based on their chemical properties such as acidity, alkalinity, ionic strength, and polarity. Samples were sealed and shaken overnight at room temperature. Finally, the desorbed materials were separated by gravity and the dye concentration was quantified by spectrophotometry. A $20 \mathrm{~mL}$ volume of co-solvent was chosen to demonstrate that these materials after being exposed to $50 \mathrm{~mL}$ of dye solutions can be recovered by using less than half of the initial volume.

\section{DATA ANALYSIS}

The amount of adsorbed BY57 onto the adsorbents was expressed as Adsorption Capacity $(q, \mathrm{mg} / \mathrm{g})$ and calculated as shown in Equation (1)

$$
q=\frac{\left(C_{i}-C_{e q}\right) \times V}{m}
$$

where $m$ is the mass of the adsorbent in grams, $V$ is the volume of the solution in $L$, and $C_{i}$ and $C_{e q}$ are the initial and at equilibrium concentrations of BY57 in $\mathrm{mg} / \mathrm{L}$.

A different way to express the adsorptive properties of a given adsorbent is Adsorption Percentage (\%ADS) where the initial and final adsorbent concentrations are compared and expressed as percentage as shown in Equation (2). Desorption percentage (\%DES) can also be calculated with a similar expression. All statistical analysis has a percent error lower than $3 \%$ as indicated by the standard deviations. $R^{2}$ and $p$-values were also used to corroborate the statistical rigor of the mathematical modeling.

$$
\% A D S=\frac{\left(C_{i}-C_{e q}\right) \times 100}{C_{i}}
$$

\section{CHARACTERIZATION OF THE ADSORBENTS}

Surface texture and morphological properties of all the tea leaves were explored by scanning electron microscopy, (SEM) using a Tabletop microscope (TM3000, HITACHI). Samples were directly observed without gold coating. 


\section{RESULTS AND DISCUSSION ADSORPTION EXPERIMENTS \\ Role of $\mathrm{pH}$ on the elimination of $\mathrm{BY} 57$}

Figure 2 displays the effect of initial $\mathrm{pH}$ on the adsorption of the dye. According to the results, higher adsorption is observed at higher $\mathrm{pH}$ values for all the adsorbents. $\mathrm{PM}$ and $\mathrm{CM}$ report an optimum $\mathrm{pH}$ at 6 and $\mathrm{CT}$ at 8 . Differences on the adsorbent composition might be responsible for this difference. CT is really not a single adsorbent; it is a mixture of black tea, and other spices like cinnamon, and cloves. Therefore, some differences on the adsorption are expected. PM and CM are more homogeneous samples, formed by tea leaves and flowers, respectively. Elimination of BY57 is greatly decreased at low $\mathrm{pH}$. This could be explained by the competition of BY57 for the adsorption sites with hydronium ions $\left(\mathrm{H}_{3} \mathrm{O}^{+}\right)$that are predominant at low $\mathrm{pH}$ values. $\mathrm{BY} 57$ is a cationic dye and therefore, it targets the same electron-rich centers, as hydronium ion does. It is important to highlight that all the adsorbents maximize their adsorption in $\mathrm{pH}$ ranging from 6 to 8. Surface and most of the contaminated waters have these $\mathrm{pH}$ values, suggesting that no previous $\mathrm{pH}$ adjustments are needed and these adsorbents can be directly used in the treatment of dye-containing waters. These materials have also shown similar behavior with other pollutants like heavy metals (Kim et al., 2013) and other dyes (Hameed, 2009; Navarro et al., 2013).

\section{Minimum adsorption mass for optimum removal}

Our main goal is to propose these adsorbents for the treatment of industrial wastewaters that contain dyes. A successful technique should result in the highest adsorption at the minimum cost. Adsorbent dose was optimized to achieve the minimum amount of spent tea leaves. As displayed in Figure 3, optimum adsorbents doses are reached with 75, 100, and $150 \mathrm{mg}$ of PM, CT, and $\mathrm{CM}$, respectively. The graph also shows that PM is the most efficient adsorbent because it maximizes the adsorption with a small mass. It would be expected that adsorption should infinitely increase with the adsorbent mass, nevertheless; a phenomenon

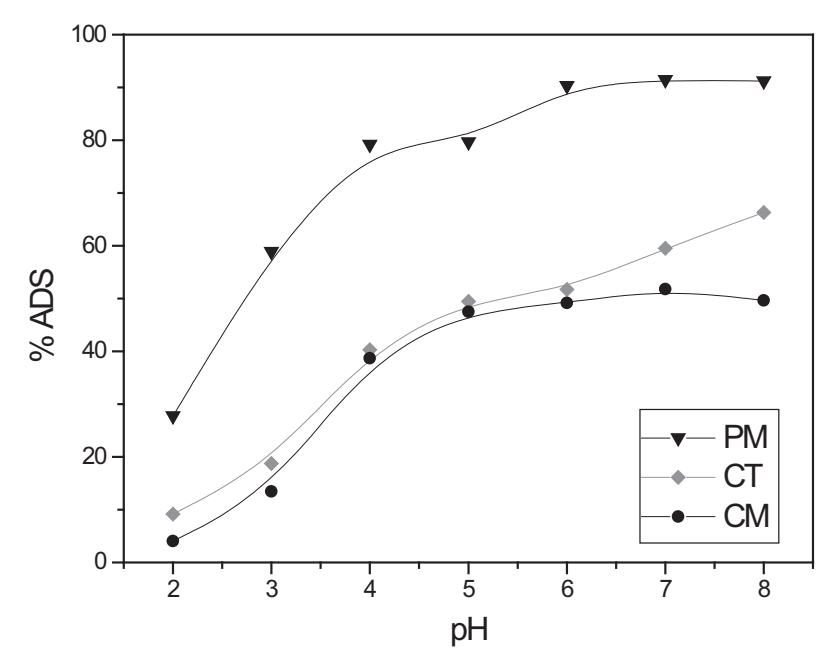

FIGURE 2 | Role of the pH on the adsorption of BY57 onto the lignocellulosic materials. called aggregation has been suggested in batch experiments. Since the adsorption happens in a sealed container, higher doses of the adsorbents are restricted to small space. This causes the formation of aggregates or clumps of the adsorbents that reduces their effective surface area. This phenomenon has also been observed in previous reports (Navarro et al., 2008a; Hameed, 2009; Dakhil, 2013; Ramesh et al., 2014).

\section{Role of the salinity and small ions}

Seawater is not exempt of chemical spills; therefore a technology that is also prepared for high-salt solutions is desired. BY57 solutions were mixed with different salt concentrations. Sodium chloride was chosen for its prevalence in seawater; sodium nitrate is a conventional salt, widely used in the evaluation of ionic strength (Navarro et al., 2008b; Liu and Wang, 2009; Iparraguirre et al., 2010), and calcium nitrate was also used to explore the effect of divalent ions on the adsorption. Results are shown in Figure 4, indicating that all the salts have a negative effect on the adsorption. Competition of cations for the adsorption is expected to be the responsible for the BY57 adsorption inhibition. The relatively smaller ionic diameters of $\mathrm{Na}^{+}$and $\mathrm{Ca}^{+2}$ might facilitate their approach to active sites. Likewise, lignocellulosic materials have a rather polar surface and will prefer polar and ionic adsorbates. Although BY57 is cationic, nevertheless; it also shows aromatic rings that reduce its polarity and therefore its affinity toward highly polar surfaces. This should promote the displacement of BY57 by small metal cations.

Figure 4 also displays that PM and CT samples are able to tolerate the effect of the salinity with $\mathrm{NaCl}$ and $\mathrm{NaNO}_{3}$. Conversely, $\mathrm{Ca}\left(\mathrm{NO}_{3}\right)_{2}$ shows a stronger effect on the adsorption of $\mathrm{PM}$ and $\mathrm{CT}$, whereas CM decreases in a lower intensity. From this data, we can hypothesize that CM adsorbs BY57 under a different mechanism that is not greatly affected by the presence of $\mathrm{Ca}^{+2}$ ions. From the results, the presence of this salts had a negative effect and therefore the highest adsorption of BY57 was achieved in their absence.

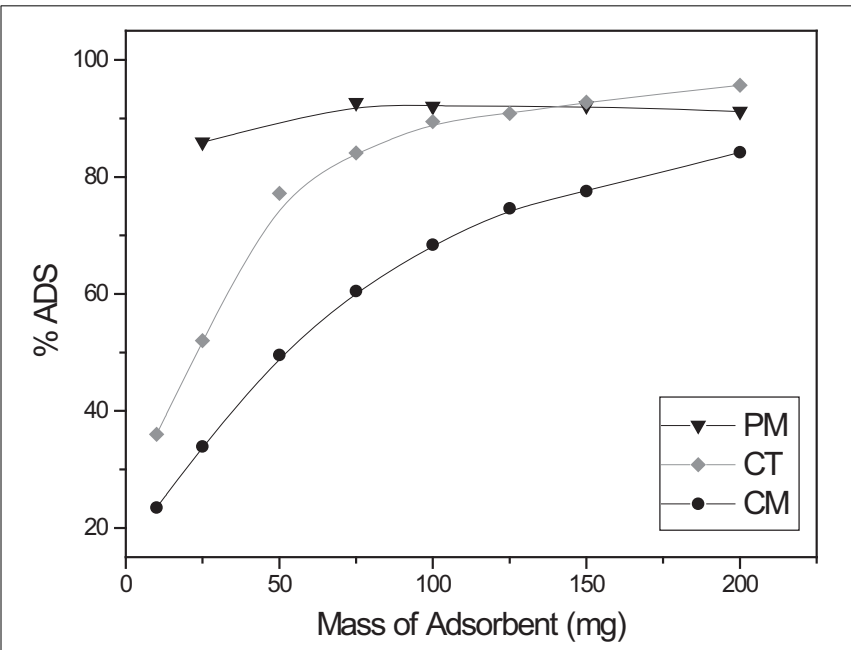

FIGURE 3 | Effect of adsorbent dosage on the adsorption of BY57. 

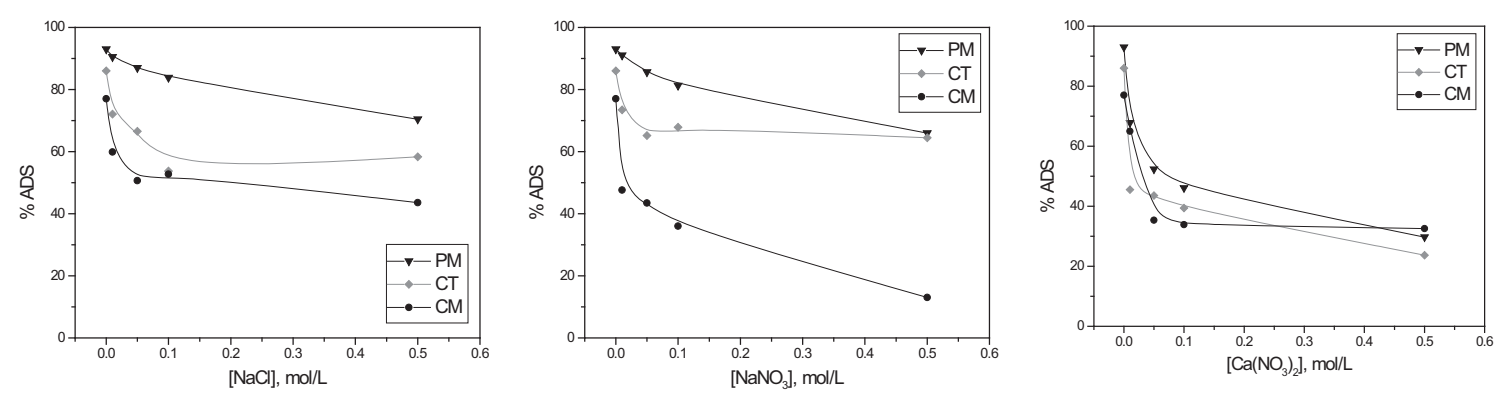

FIGURE 4 | Role of salinity on the adsorption of BY57 onto tea leaves.

\section{Crowding agents as covalent interferences}

The effect of ionic strength addresses the interference of ionic substances in solutions. However, there are other compounds also present in contaminated waters like surfactants, proteins, soluble carbohydrates, oils, and microscopic organisms. Even though these substances are not competing for the same adsorption sites as the adsorbate, but their presence could alter the adsorption removal of BY57. PEG is an inert polymers and highly soluble in water. Preliminary experiments showed that PEG does not adsorb onto the tea leaves and does not adsorb BY57. It is a very stable polymer that remains in solution. As a polyether, PEG, does not form strong intermolecular forces, other than dispersion or very weak dipole-dipoles. BY57 solutions were mixed with different PEG solutions to test its effect on the adsorption. The experimental results are shown in Figure 5. From the graph, CT is greatly affect by the presence of PEG, whereas adsorption onto PM and CM remained constant to PEG concentrations are high as $10 \%$ $\mathrm{m} / \mathrm{v}$. CT might be more sensitive to the presence of PEG due to the heterogeneity of its composition. PM and CM are able to tolerate the presence of PEG, demonstrating their applicability in the presence of covalent impurities. Another interpretation of the results could be the exposure of adsorption sites in PM and CM that does not reflect major disadvantages with crowding agents. CT might have hindered adsorption sites have encounter difficulties to approach BY57 for its adsorption.

\section{Effect of heavy metals and large divalent cations}

Contaminated wastewaters also contain large cations in solution (heavy metals). Copper and zinc are commonly found in industrial waters, and both have shown to be of environmental importance and associated with serious health consequences (Liu and Wang, 2009; Kotrba et al., 2011). The presence of these heavy metals and their effect on the adsorption of BY57 are reported in Figure 6. Experimental results indicate a nonlinear correlation between heavy metals and the adsorption of BY57. The observed changes are not substantial and could be considered negligible. Therefore, it could be concluded that our adsorbents are able to eliminate BY57 dye even in the presence of other pollutants like copper and zinc ions. These results do not demonstrate that tea leaves prefer BY57, because heavy metals could be adsorbed with a totally different mechanism and even using different adsorption sites. What it is important to point out is that large ions like copper and zinc do not block the adsorption or compete with BY57 as sodium and calcium ions do. This discrepancy could also

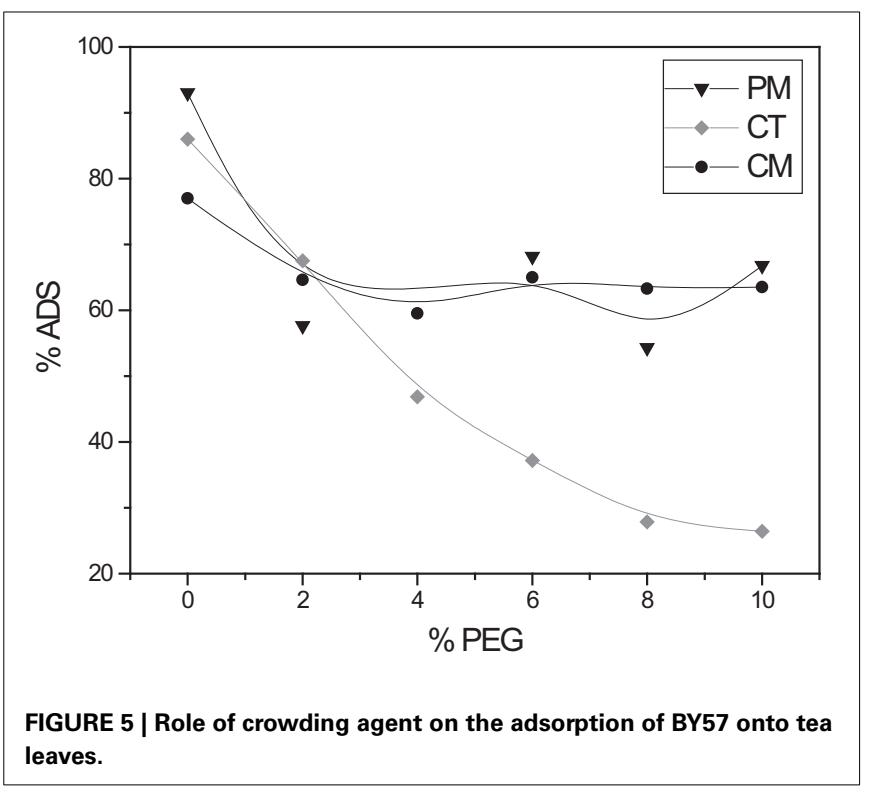

be associated with the mass/charge ratio of calcium and sodium, compared to copper and zinc. These heavy metals are called soft acids, as they dissipate the positive charge in a large ionic volume, and decrease their Lewis acid capacity. Lead nitrate was only studied for CT, since zinc nitrate precipitated at the optimum $\mathrm{pH}$ value (8) for this particular adsorbent. Copper remained soluble at $\mathrm{pH} 6$ and 8 for all the adsorbents and zinc was soluble at pH 6 , for PM and CM; for these particular metal concentrations.

\section{Isotherm modeling and maximum adsorption capacity}

Adsorption isotherms are mathematical models that allow us to determine important equilibrium parameters such as maximum adsorption capacity, adsorption affinity, equilibrium constant, and adsorption intensity. Two isotherms were tested for their ability to describe the experimental results, namely Langmuir and Freundlich isotherms (Liu and Wang, 2009; Kotrba et al., 2011). Linear regression was used to fit all the data points. Correlation coefficient and p-values were determined as statistical significance testing. As shown in Figure 7, the adsorption capacities follow the trend PM >CT > CM, same as the previously studied effects. A given mass of adsorbent has a finite number of adsorption sites that are totally saturated at a particular pollutant concentration. 

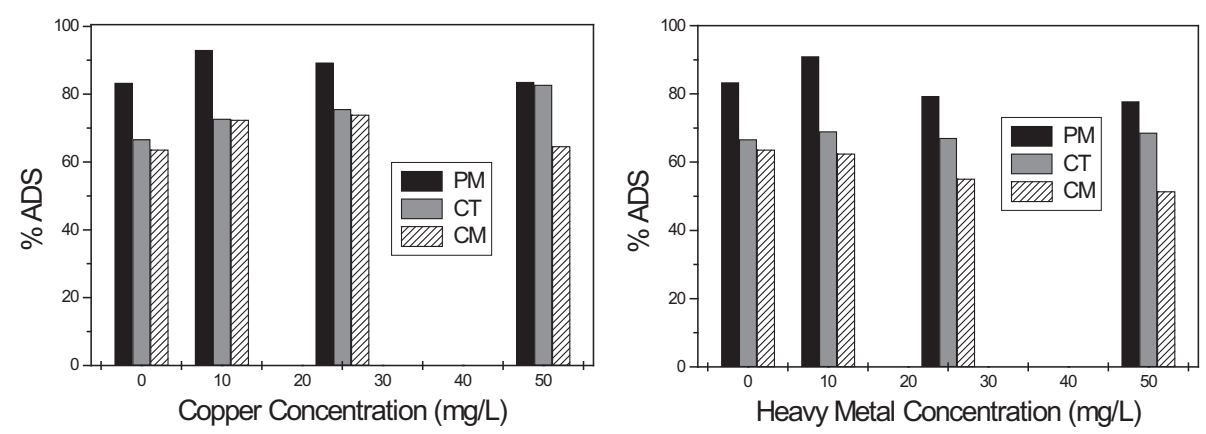

FIGURE 6 | Effect of large heavy metal cations on the adsorption of BY57. Copper effect (left) and zinc and lead effect (right).

Table 1 | Langmuir and Freundlich Isotherm Constants for the adsorption of BY57 onto lignocellulosic adsorbents.

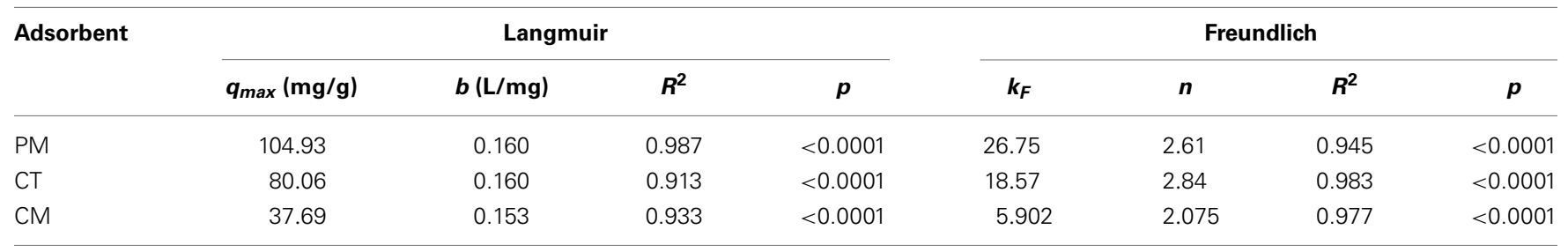

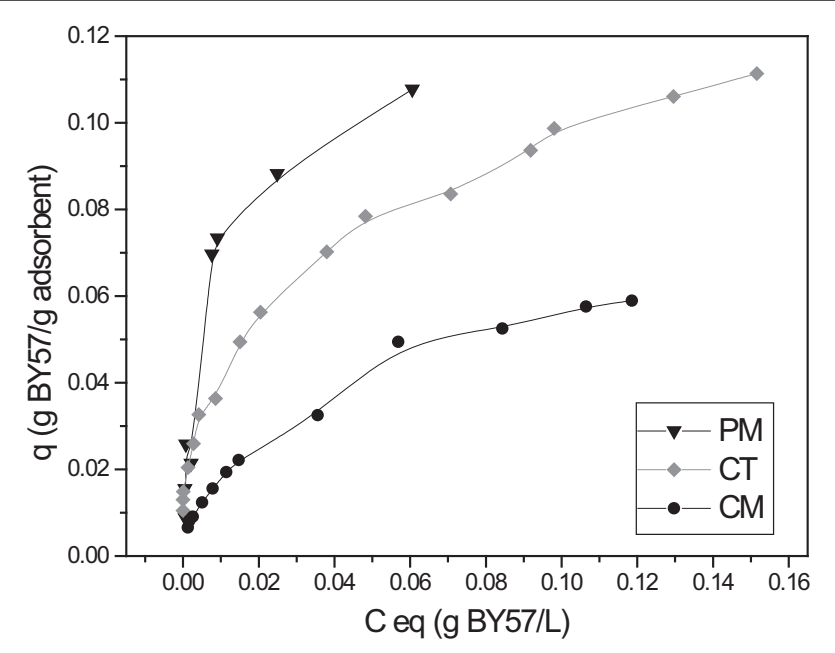

FIGURE 7 | Adsorption Isotherm of the adsorption of BY57 onto tea leaves.

Langmuir theory assumes uniform adsorption energy on the surface of the adsorbent, where the migration of the pollutant among neighboring active sites is restricted (Volesky, 2004; Liu and Wang, 2009). The mathematical expression of the Langmuir isotherm is calculated as follows (Equation 3):

$$
q=\frac{q_{\max } \times b \times C_{e q}}{1+b \times C_{e q}}
$$

where $q_{\max }(\mathrm{mg} / \mathrm{g})$ and $b(\mathrm{~L} / \mathrm{mg})$ are the Langmuir constants related to the maximum adsorption capacity and to the adsorption energy, respectively. The $q_{\max }$ constant represents the total number of available adsorption sites for one adsorbent and is commonly used to compare adsorbents based on their ability to remove pollutants. The $b$ constant is also important because it allows us to compare the adsorption efficiency between two different adsorbents under the same experimental conditions. A higher $b$ constant means a higher adsorbent/pollutant affinity. The corresponding calculated Langmuir constants are shown in Table 1.

The data was also analyzed by the model of Freundlich that is derived assuming a logarithmic decrease in the enthalpy of adsorption with the increase in the fraction of occupied sites and based on sorption on heterogeneous surfaces (Volesky, 2004; Kotrba et al., 2011). Freundlich's expression is given by Equation (4):

$$
q=k_{F} \times C_{e q}^{1 / n}
$$

where $k_{F}$ and $n$ are the Freundlich constant, related to the adsorption capacity and the adsorption intensity, respectively. If $n=1$, then the partition between the two phases are independent of the concentration. If $n>1$, it indicates a normal adsorption, whereas $n<1$ suggests cooperative adsorption (Liu and Wang, 2009; Kim et al., 2013; Navarro et al., 2013).

Isotherm modeling results are summarized in Table 1. From the results, the highest adsorption is observed with PM, reaching up to $105 \mathrm{mg}$ of BY57 per gram of adsorbent. Conversely, CM is the poorest adsorbent, with only $38 \mathrm{mg}$ of BY57 per gram of CM. Other lignocellulosic materials have been studied as dye adsorbents and obtained similar results. Dakhil has recently adsorbed methylene blue using spent tea leaves and obtained a $q_{\max }$ of $46 \mathrm{mg} / \mathrm{g}$ (Dakhil, 2013). Ramesh also used spent tea dust to remove methylene blue, obtaining a $q_{\max }$ of $86 \mathrm{mg} / \mathrm{g}$ (Ramesh et al., 2014). Finally, Prasad and Santhi worked with Acacia nilotica leaves to eliminate crystal violet and rhodamine B, reporting 
$q_{\max }$ values of 33 and $37 \mathrm{mg} / \mathrm{g}$, respectively (Prasad and Santhi, 2012). Our research group has also explored the adsorption using spent green tea leaves as adsorbents, reporting $q_{\max }$ values around $50 \mathrm{mg} / \mathrm{g}$ (unpublished results). On the other hand, the $b$ Langmuir constant indicates a relatively similar adsorbent/dye affinity for the three adsorbents. Hameed (2009), Dakhil (2013), and Ramesh et al. (2014) reported $b$ constant values of 0.0812 , 0.0153 , and $0.26 \mathrm{~L} / \mathrm{mg}$, respectively with their dyes.

As for the Freundlich's model, equilibrium constant $k_{F}$ shows the following adsorption trend: $\mathrm{PM}>\mathrm{CT}>\mathrm{CM}$, corroborating the results obtained with Langmuir's theory. The $n$ constant also demonstrates favorable adsorptions for the three adsorbent with similar intensities. Finally, statistical significance indicates that both, Langmuir and Freundlich models fit the adsorption of BY57 onto the three adsorbents, suggesting a combined adsorption mechanism (Volesky, 2004).

\section{DESORPTION EXPERIMENTS}

A successful technique for the decolorization of hair dyes and colorants should be practical and economical under any experimental conditions. Spent tea leaves are advantageous because they are massively produced by industries that produce bottled tea drink and become a waste disposal concern. One of the most important drawbacks of the commonly used techniques, is the generation of sludge or byproducts that need to be taken care of and even require additional expenses or are more toxic than the original pollutants (Liu and Wang, 2009; Kotrba et al., 2011). The recyclability of adsorbents is an important factor to consider when choosing the most appropriate materials. For this purpose, different diluted solutions were used to desorb BY57 and recover the adsorbent. The main goal is to recycle the adsorbent for continuous process several times with the same adsorption properties. These co-solvents were chosen based on their chemical properties including polarity, ionic strength, acidity, and alkalinity. Figure 8 displays the desorption of BY57 from the lignocellulosic materials with these co-solvents. The results indicate that diluted $\mathrm{HCl}(0.1 \mathrm{M})$ solution is the most effective desorbing solution, reaching values close to $70 \%$ for all the adsorbents. Crucial and important results were obtained with distilled water, where almost null desorption is observed. This means that these materials can be used in the preparation of filters for large scale purification because clean water cannot get re-contaminated by the discharge of the adsorbed dye.

\section{CHARACTERIZATION OF THE ADSORBENTS BY MORPHOLOGY AND TEXTURE}

Figure 9 shows the scanning electron micrographs of the three lignocellulosic adsorbents and their textural and morphological properties. All the images indicate highly porous surfaces and structures that are typically seen in plants. They all have heterogeneous and random morphologies that are expected in adsorbents. These imperfections on the surface are able to house and trap pollutants by interactions with the adsorption sites. PM shows a more organized structure, with veins and fibers, whereas CT is more random, due to its composition and presence of cinnamon, cloves, and other herbs. CM is a more special structure, because it comes from flowers. CM is also porous but its pores have bigger diameters when compared to CT and PM. These images could

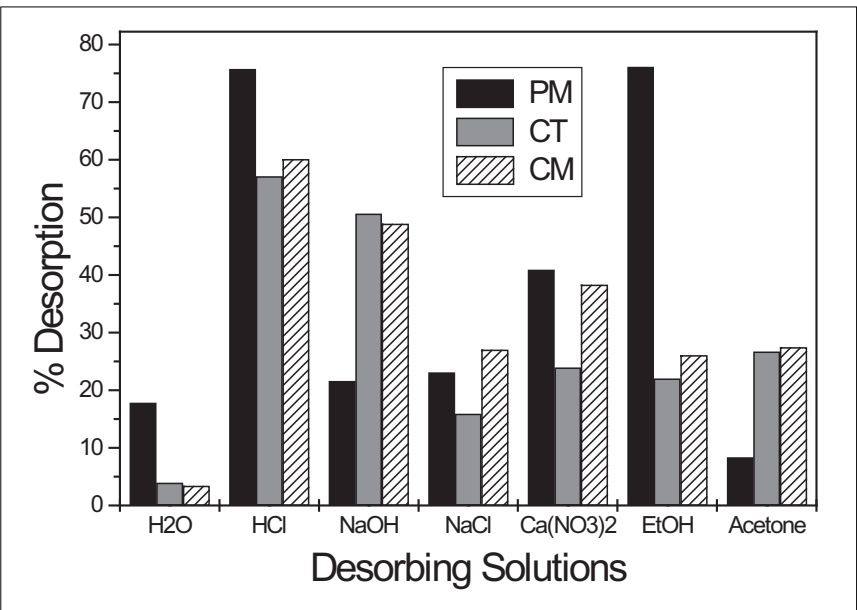

FIGURE 8 | Desorption of BY57 from the lignocellulosic adsorbents.

be associated with the adsorption results, as PM has a heterogeneous, yet organized, surface and also reports the best adsorption. CT has also heterogeneous surface, but less than PM. CT reports lower adsorption, but we believe it is because of its composition and weaker adsorption sites that might be present. Finally, CM has a surface that has low heterogeneity and its porosity is not optimum for the adsorption of BY57.

\section{CONCLUSIONS}

Spent tea residues of PM, CT, and CM were used as raw adsorbents for the elimination of the hair dye BY57 from aqueous solutions. Lignocellulosic structure and highly polar functional groups are potential adsorption sites for BY57. Batch experiments were tested to investigate the optimum equilibrium conditions at room temperature. The adsorption capacity followed the trend: $\mathrm{PM}>\mathrm{CT}>\mathrm{CM}$. The maximum adsorption was achieved at $\mathrm{pH}$ 6 , with a minimum mass of $75 \mathrm{mg}$ of PM and in the absence of salts, crowding agents, and heavy metals. Ionic strength has a negative effect on the adsorption by disruption of polar and electrostatic interaction between the dye and the adsorbent, and also due to the competition of small ions for the adsorption sites. PEG was used as a crowding agent to mimic covalent impurities. Results indicate small effect on the adsorption with the exception of CT. This indicates that these adsorbents can be used in solution with high content of bulky impurities, including proteins, oils, and other molecules. Adsorption isotherms were modeled according to Langmuir and Freundlich theories, reporting a maximum adsorption capacity for PM, CT, and CM of 105, 80, and $28 \mathrm{mg}$ of BY57 per gram of adsorbent. Heavy metals like copper and zinc ions were used as secondary pollutants to evaluate their effect in the removal of BY57. The observed experimental data suggests that these adsorbents are not affected by the presence of heavy metals mostly likely due to differences in the adsorption mechanism or targeted active sites. SEM analyses demonstrate that these lignocellulosic materials possess a heterogeneous surface with pockets and protrusions that are optimum adsorption sites for pollutants. Finally, desorption experiments indicate that a diluted solution of $\mathrm{HCl}$ is able to desorb up to $70 \%$ of BY57 from the adsorbent and allows its reutilization in consecutive adsorption cycles. This study clearly shows that raw adsorbents are 

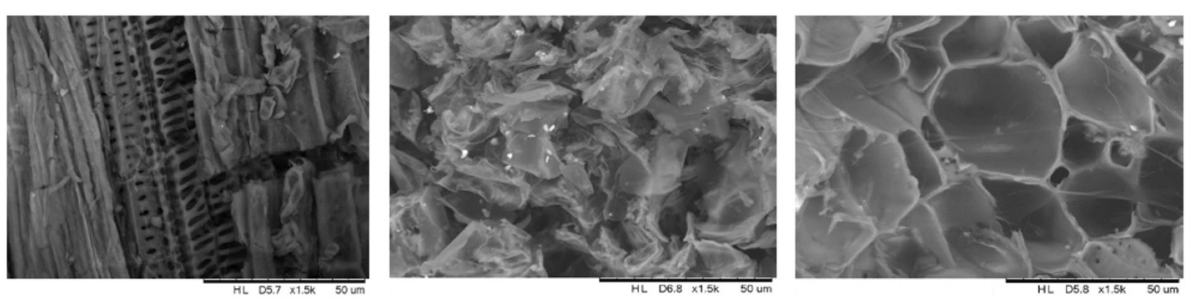

FIGURE 9 | Scanning electron microscopy analysis of the adsorbents: PM (left), CT (middle), and CM (right).

cost-effective and "green" materials with potential for the removal of dyes from contaminated waters. We expect this study will start a new lead in the development of new eco-friendly alternatives for the elimination of organic and inorganic contaminants.

\section{ACKNOWLEDGMENTS}

This research was conducted thanks to the financial support of L'Oreal and CONCYTEC (Peru) under Project 001-2012-L'Oreal. Abel E. Navarro was also supported by the Science Department and CSTEP at BMCC. Chiu Hong Lee is greatly thanked for her technical assistance.

\section{REFERENCES}

Cuizano, N., Llanos, B., and Navarro, A. E. (2009). Environmental applications of adsorption by natural biopolymers. part I - phenolic compounds. Rev. Soc. Quim. Perú. 75, 488-494. Available online at: http://www.scielo. org.pe/scielo.php?script=sci_abstract\&pid=S1810-634X2009000400012\&lng= es\&nrm=iso\&tlng=en

Dakhil, I. (2013). Adsorption of methylene blue dye from wastewater by spent tea leaves. J. Kerbala Univ. 11, 5-14. Available online at: http://www.iasj.net/iasj?func=fulltext\&aId $=78970$

Diaz, C., Jacinto, C., Medina, R., Navarro, A., Cuizano, N., and Llanos, B. (2013). Study of the biosorption of chromium (VI) on cross-linked quaternary chitosan for their application on the bioremediation of wastewaters. Rev. Soc. Quim. Perú. 79, 304-318. Available online at: http://www.scielo.org.pe/pdf/rsqp/v79n4/a03v79n4.pdf

Easton, J. (1995). The Dye Maker's View. Nothingham: Society of dyers and colourist.

Gonzalez, M., and Fuentes, L. (2009). Degradation of immobilized azo dyes by Klebsiella sp. UAP-b5 isolated from maize bioadsorbent. J. Hazard. Mater. 161, 769-774. doi: 10.1016/j.jhazmat.2008.04.023

Guo, M., Lu, F., Liu, M., Li, T., Pu, J., Wang, N., et al. (2008). Purification of recombinant laccase from Trametes versicolor in Pichia methanolica and its use for the decolorization of anthraquinone dye. Biotechnol. Lett. 30, 2091-2096. doi: 10.1007/s10529-008-9817-z

Hameed, B. (2009). Spent tea leaves: a new non-conventional and low-cost adsorbent for removal of basic dye from aqueous solutions. J. Hazard. Mater. 161, 753-759. doi: 10.1016/j.jhazmat.2008.04.019

Iparraguirre, E., Manrique, A., Navarro, A., Cuizano, N., Medina, R., and Llanos, B. (2010). Biosorption of gold (III) by means of quaternary and cross-linked quaternary chitosan. Rev. Soc. Quim. Perú. 76, 355-365. Available online at: http://www.scielo.org.pe/pdf/rsqp/v76n4/a06v76n4.pdf

Kim, T., Yang, D., Kim, J., Musaev, H., and Navarro, A. (2013). Comparative adsorption of highly porous and raw adsorbents for the elimination of copper (II) ions from wastewaters. Trends Chromatog. 8, 97-108. Available online at: http://journal.frontiersin.org/Journal/10.3389/fenvs.2014.00028/pdf

Kotrba, P., Mackova, M., and Macek, T. (2011). Microbial Biosorption of Metals. New York, NY: Springer Science. doi: 10.1007/978-94-007-0443-5

Liu, Y., and Wang, J. (2009). Fundamental and Applications of Biosorption Isotherms, Kinetics, and Thermodynamics. New York, NY: Nova Science Publishers.

Naidoo, M., Kostadinova, R., Zahir, H., and Navarro, A. (2013). "Chai tea as a potential adsorbent of dyes from wastewaters," in Proceedings of 2 nd
International Conference on Earth Science and Climate Change, J. Earth Sci. Climate Change, Vol. 4, (Las Vegas, NV), 4.

Navarro, A., Chang, E., Chang, P., Yoon, S., and Manrique, A. (2013). Separation of dyes from aqueous systems by magnetic alginate beads. Trends Chromatog. 8, 31-41. Available online at: http://www.google.com/ url? $\mathrm{sa}=\mathrm{t} \& \mathrm{rct}=\mathrm{j} \& \mathrm{q}=\&$ esrc $=\mathrm{s} \&$ source $=$ web $\& \mathrm{~cd}=1 \& \mathrm{ved}=0 \mathrm{CCIQFjAA \& url}=\mathrm{http} \%$ 3A\%2F\%2Fwww.researchgate.net\%2Fprofile\%2FAbel_Navarro\%2Fpublication \%2F261135723_Separation_of_Dyes_from_aqueous_solutions_by_magnetic_ alginate_beads\%2Ffile\%2F72e7e533c6aa3a854f.pdf\&ei=tvKqU8LWErK0sQS9 qoGIBA\&usg=AFQjCNGp_P5SFTSPEIzYbUOyPg5eb1KqIg\&bvm=bv.696200 78,d.cWc

Navarro, A., Cuizano, N., Portales, R., and Llanos, B. (2008b). Adsorptive removal og 2-nitrphenol and 2-chlorophenol by cross-linked algae from aqueous solutions. Sep. Sci. Technol. 43, 3183-3199. doi: 10.1080/01496390802221642

Navarro, A., Portales, R., Sun-Kou, R., and Llanos, B. (2008a). Effect of pH on phenol biosorption by seaweeds. J. Hazard. Mater. 156, 405-411. doi: 10.1016/j.jhazmat.2007.12.039

Prasad, A., and Santhi, T. (2012). Adsorption of hazardous cationic dyes from aqueous solution onto Acacia nilotica leaves as an eco friendly adsorbent. Sustain. Environ. Res. 22, 113-122. Available online at: http://ser.cienve.org.tw/download/22-2/22-2-7.pdf

Ramesh, S., Gandhimathi, R., Elavarasi, T., Isai, T., Sowmya, K., and Nidheesh, P. (2014). Comparison of methylene blue adsorption from aqueous solution using spent tea dust and raw coir pith. Global NEST J. 16, 146-159.

Robinson, T., Mcmullan, G., Marchant, R., and Nigman, P. (2001). Remediation of dyes in textile effluent: a critical review on current treatment technologies with a proposed alternative. Bioresour. Technol., 77, 247-255. doi: 10.1016/S09608524(00)00080-8

Singh-Rai, H., Shankar, M., Singh, J., Bansal, T., Vats, P., and Banerjee, U. (2005). Removal of dyes from the effluent of textile an dyestuff manufacturing industry: a review of emerging techniques with reference to biological treatment. Crit. Rev. Environ. Sci. Technol. 35, 219-238. doi: 10.1080/10643380590917932

Volesky, B. (2004). Sorption and Biosorption. Montreal; St. Lambert, QC: BV SORBEX Inc.

Zollinger, H. (1991). Color Chemistry-Synthesis, Properties and Applications of Organic Dyes and Pigments. New York, NY: Vch Publications.

Conflict of Interest Statement: The authors declare that the research was conducted in the absence of any commercial or financial relationships that could be construed as a potential conflict of interest.

Received: 22 April 2014; accepted: 13 June 2014; published online: 04 July 2014. Citation: Zahir H, Naidoo M, Kostadinova R-M, Ortiz KA, Sun-Kou R and Navarro AE (2014) Decolorization of hair dye by lignocellulosic waste materials from contaminated waters. Front. Environ. Sci. 2:28. doi: 10.3389/fenvs.2014.00028

This article was submitted to Environmental Toxicology, a section of the journal Frontiers in Environmental Science.

Copyright (c) 2014 Zahir, Naidoo, Kostadinova, Ortiz, Sun-Kou and Navarro. This is an open-access article distributed under the terms of the Creative Commons Attribution License (CC BY). The use, distribution or reproduction in other forums is permitted, provided the original author(s) or licensor are credited and that the original publication in this journal is cited, in accordance with accepted academic practice. No use, distribution or reproduction is permitted which does not comply with these terms. 